\title{
Search for neutrino charged current coherent pion production in SciBooNE
}

\author{
K. Hiraide $(*)$ for the SciBoone Collaboration \\ Department of Physics, Kyoto University - Kyoto 606-8502, Japan
}

(ricevuto il 10 Novembre 2009; pubblicato online il 20 Gennaio 2010)

\begin{abstract}
Summary. - SciBooNE is a neutrino experiment measuring the neutrino cross-sections on carbon in the one $\mathrm{GeV}$ region. We have performed a search for charged current coherent pion production from muon neutrinos scattering on carbon, $\nu_{\mu}{ }^{12} \mathrm{C} \rightarrow \mu^{-12} \mathrm{C} \pi^{+}$. No evidence for coherent pion production is observed. We set $90 \%$ confidence level upper limits on the cross-section ratio of charged current coherent pion production to the total charged current cross-section at $0.67 \times 10^{-2}$ at mean neutrino energy $1.1 \mathrm{GeV}$ and $1.36 \times 10^{-2}$ at mean neutrino energy $2.2 \mathrm{GeV}$.

PACS 13.15.+g - Neutrino interactions.

PACS 13.60.Le - Meson production.

PACS 25.30.Pt - Neutrino-induced reactions.

PACS 95.55.Vj - Neutrino, muon, pion, and other elementary particle detectors; cosmic ray detectors.
\end{abstract}

\section{1. - Introduction}

Evidences of neutrino oscillation have been observed by several experiments over the last decade, and neutrino oscillation physics has entered the era of precision measurements. In the second generation accelerator neutrino oscillation experiments, precise knowledge of neutrino cross-sections with nuclei are important to achieve the best sensitivity. However, the typical accuracy of neutrino-nucleus cross-sections in the one GeV region, which is relevant to many future experiments, is $20 \%$ with our current knowledge. Among several neutrino interaction channels, neutrino interactions producing single pion form significant backgrounds for neutrino oscillation studies with a few-GeV neutrino beam, and thus understanding those processes is essential. It has been known for years that neutrinos can produce pions by interacting coherently with the nucleons forming the target nucleus. Both charged current (CC) and neutral current (NC) coherent modes

$\left(^{*}\right)$ Present address: Kamioka Observatory, Institute for Cosmic Ray Research, University of Tokyo, Gifu 506-1205, Japan.

(C) Società Italiana di Fisica / INFN - Laboratori Nazionali di Frascati 


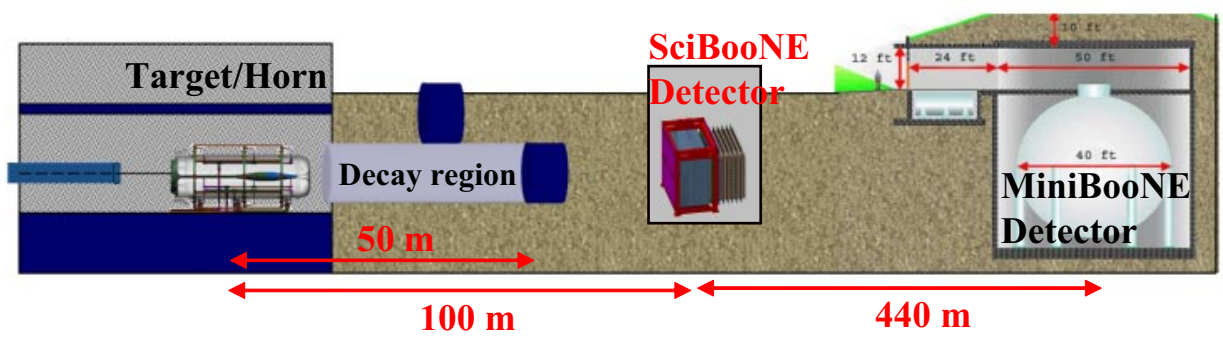

Fig. 1. - Schematic drawing of the experimental setup of SciBooNE.

are possible, $\nu_{\mu} A \rightarrow \mu^{-} A \pi^{+}$and $\nu_{\mu} A \rightarrow \nu_{\mu} A \pi^{0}$, where $A$ is a nucleus. The outgoing lepton and pion tend to go in the forward direction in the lab frame, and no nuclear breakup occurs. Recent results on coherent pion production have induced interest of the neutrino physics community. The non-existence of charged current coherent pion production in a $1.3 \mathrm{GeV}$ wide-band neutrino beam has been reported by $\mathrm{K} 2 \mathrm{~K}$ [1], while there exist charged current coherent pion production positive results at higher neutrino energies. On the one hand, evidence for $\mathrm{NC}$ coherent pion production in the similar neutrino energy has been recently reported by MiniBooNE [2].

The SciBooNE experiment [3] is designed to measure the neutrino cross sections on carbon in the one $\mathrm{GeV}$ region. In this paper, we report the first measurement of charged current coherent pion production on carbon by neutrinos in the SciBooNE experiment, which was recently published in ref. [4].

\section{2. - The SciBooNE experiment}

Figure 1 shows a schematic drawing of the experimental setup of SciBooNE. The experiment uses the Booster Neutrino Beam (BNB) at Fermilab. The primary proton beam, with kinetic energy $8 \mathrm{GeV}$, is extracted to strike a $71 \mathrm{~cm}$ long, $1 \mathrm{~cm}$ diameter beryllium target. Each beam spill consists of 81 bunches of protons, containing typically $4 \times 10^{12}$ protons in a total spill duration of $1.6 \mu \mathrm{s}$. The target sits at the upstream end of a magnetic focusing horn that is pulsed with approximately $170 \mathrm{kA}$ to focus the mesons, primarily $\pi^{+}$, produced by the $p$-Be interactions. In a $50 \mathrm{~m}$ long decay pipe following the horn, $\pi^{+}$decay and produce neutrinos, before the mesons encounter an absorber. The flux is dominated by muon neutrinos ( $93 \%$ of total), with small contributions from muon antineutrinos (6.4\%), and electron neutrinos and antineutrinos ( $0.6 \%$ in total). The flux-averaged mean neutrino energy is $0.7 \mathrm{GeV}$. When the horn polarity is reversed, $\pi^{-}$are focused and hence a predominantly antineutrino beam is created.

The SciBooNE detector is located $100 \mathrm{~m}$ downstream from the neutrino production target. The detector complex consists of three sub-detectors: a fully active fine-grained scintillator tracking detector (SciBar), an electromagnetic calorimeter (EC) and a muon range detector (MRD). The SciBar detector consists of 14336 extruded plastic scintillator strips, each $1.3 \times 2.5 \times 300 \mathrm{~cm}^{3}$. The scintillators are arranged vertically and horizontally to construct a $3 \times 3 \times 1.7 \mathrm{~m}^{3}$ volume with a total mass of 15 tons. Each strip is read out by a wavelength-shifting fiber attached to a 64-channel multi-anode PMT. Charge and timing information from each MA-PMT is recorded by custom electronics. The minimum length of a reconstructed track is $8 \mathrm{~cm}$ which corresponds to a proton with momentum of $450 \mathrm{MeV} / c$. The EC is installed downstream of SciBar, and consists of 
32 vertical and 32 horizontal modules made of scintillating fibers embedded in lead foils. Each module has dimensions of $4.0 \times 8.2 \times 262 \mathrm{~cm}^{3}$, and is read out by two $1^{\prime \prime}$ PMTs on both ends. The EC has a thickness of $11 X_{0}$ along the beam direction to measure $\pi^{0}$ emitted from neutrino interactions and the intrinsic $\nu_{e}$ contamination. The energy resolution is $14 \% / \sqrt{E(\mathrm{GeV})}$. The MRD is located downstream of the EC in order to measure the momentum of muons up to $1.2 \mathrm{GeV} / c$ with range. It consists of 12 layers of $2^{\prime \prime}$-thick iron plates sandwiched between layers of $6 \mathrm{~mm}$-thick plastic scintillator planes. The cross-sectional area of each plate is $305 \times 274 \mathrm{~cm}^{2}$. The horizontal and vertical scintillator planes are arranged alternately, and the total number of scintillators is 362 .

The experiment took both neutrino and antineutrino data from June 2007 until August 2008. In total, $2.64 \times 10^{20} \mathrm{POT}$ were delivered to the beryllium target during the SciBooNE data run. After beam and detector quality cuts, $2.52 \times 10^{20} \mathrm{POT}$ are usable for physics analyses; $0.99 \times 10^{20} \mathrm{POT}$ for neutrino data and $1.53 \times 10^{20} \mathrm{POT}$ for antineutrino data. Results from the full neutrino data sample are presented in this paper.

\section{3. - Event selection}

The experimental signature of charged current coherent pion production is the existence of two and only two tracks originating from a common vertex, both consistent with minimum ionizing particles (a muon and a charged pion).

To identify charged current events, we search for tracks in SciBar matching with a track or hits in the MRD. Such a track is defined as a SciBar-MRD matched track. The most energetic SciBar-MRD matched track in any event is considered as the muon candidate. The matching criteria impose a muon momentum threshold of $350 \mathrm{MeV} / c$. The neutrino interaction vertex is reconstructed as the upstream edge of the muon candidate. We select events whose vertices are in the SciBar fiducial volume, $2.6 \mathrm{~m} \times 2.6 \mathrm{~m} \times 1.55 \mathrm{~m}$, a total mass of 10.6 tons. Finally, event timing is required to be within $2 \mu$ s beam timing window. The cosmic-ray background contamination in the beam timing window is only $0.5 \%$, estimated using a beam-off timing window. Approximately 30000 events are selected as our standard charged current sample, which is called SciBar-MRD matched sample. According to the MC simulation, the selection efficiency and purity of true $\nu_{\mu}$ charged current events are $27.9 \%$ and $92.8 \%$, respectively. Two subsamples of the SciBar-MRD matched sample are further defined: the MRD stopped sample and the MRD penetrated sample. Events with the muon stopping in the MRD are classified as MRD stopped events. Events with the muon exiting from the downstream end of the MRD are defined as the MRD penetrated sample, in which we can measure only a part of the muon momentum. The average neutrino beam energy for true charged current events in the MRD stopped and MRD penetrated samples is $1.0 \mathrm{GeV}$ and $2.0 \mathrm{GeV}$, respectively, enabling a measurement of charged current coherent pion production at two different mean neutrino energies.

Once the muon candidate and the neutrino interaction vertex are reconstructed, we search for other tracks originating from the vertex. Most events are reconstructed as either one track or two track events. The two-track sample is further divided based on particle identification. The particle identification variable, Muon Confidence Level $(\mathrm{MuCL})$ is related to the probability that a particle is a minimum ionizing particle based on the energy deposition. The probability of misidentification is estimated to be $1.1 \%$ for muons and $12 \%$ for protons. We first require that the MuCL of the SciBar-MRD matched track is greater than 0.05 to reject events with a proton penetrating into the MRD. Then the second track in the event is classified as a pion-like or a proton-like track 


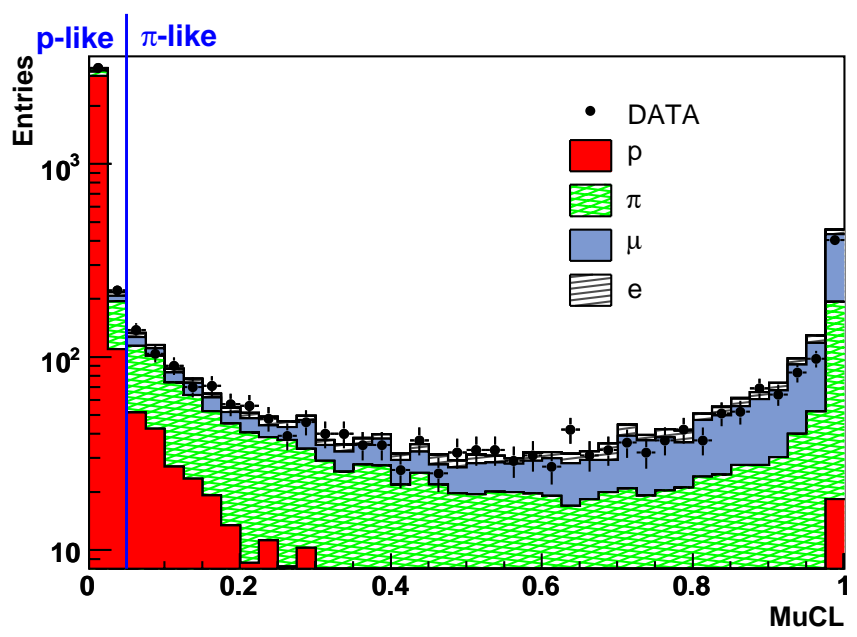

Fig. 2. - MuCL of the second track for two-track events in the MRD stopped sample.

with the same MuCL threshold. Figure 2 shows the contributions to the second track from true proton, pion, muon, and electron tracks as predicted by the MC simulation.

In a charged current resonant pion event, $\nu p \rightarrow \mu^{-} p \pi^{+}$, the proton is often not reconstructed due to its low energy, and such an event is therefore identified as a two-track $\mu+\pi$ event. To separate charged current coherent pion events from charged current resonant pion events, additional protons with momentum below the tracking threshold are instead detected by their large energy deposition around the vertex, so-called vertex activity. Figure 3 shows the maximum energy for $\mu+\pi$ events in the MRD stopped sample. A peak around $6 \mathrm{MeV}$ corresponds to the energy deposited in the strip containing

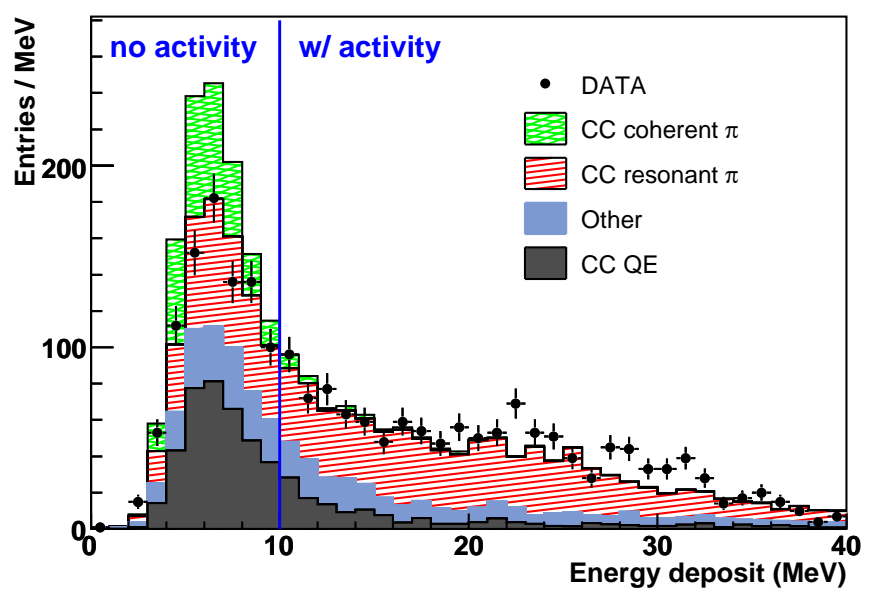

Fig. 3. - Maximum deposited energy in a strip around the vertex for the $\mu+\pi$ events in the MRD stopped sample. 

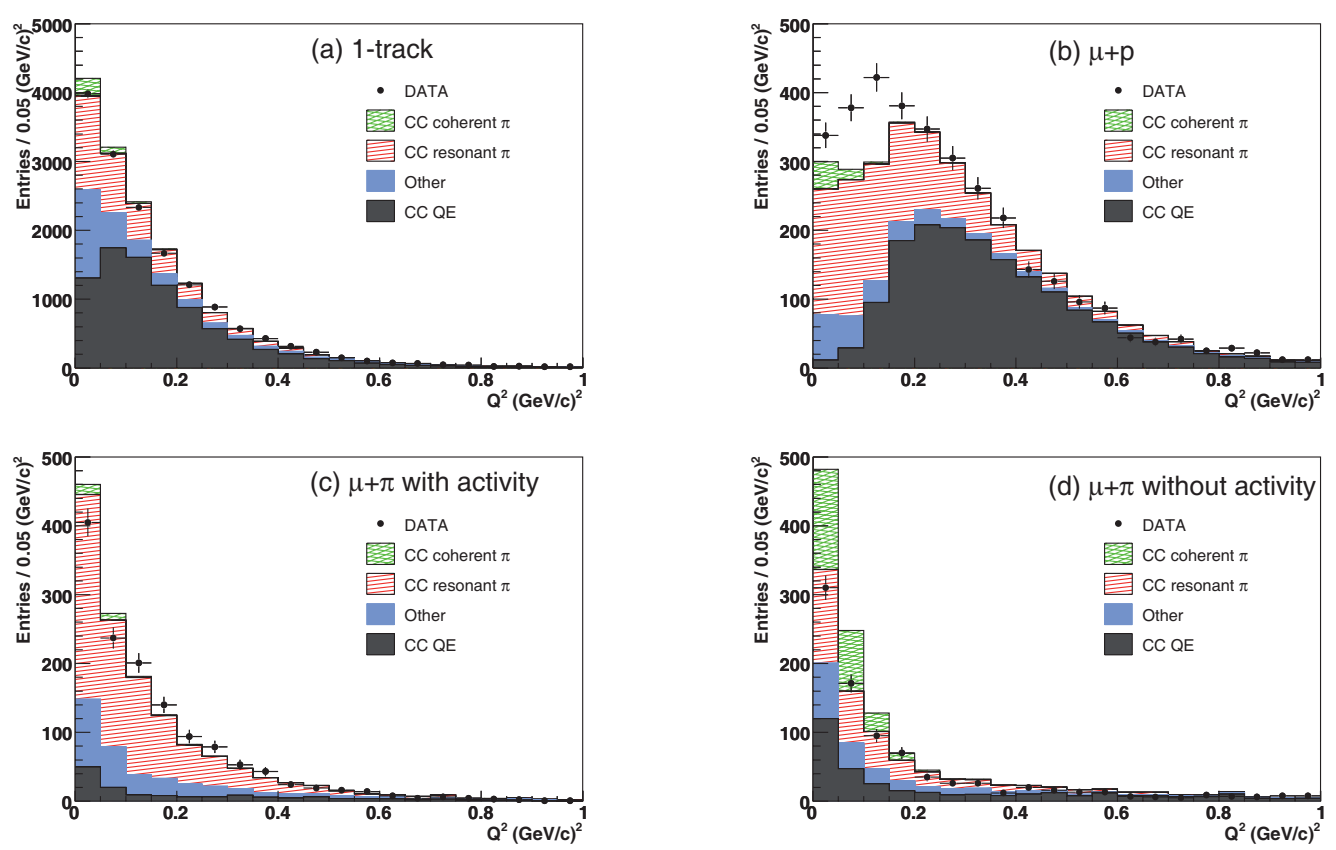

Fig. 4. - Reconstructed $Q^{2}$ after fitting for (a) the one track, (b) $\mu+p$, (c) $\mu+\pi$ with activity, and (d) $\mu+\pi$ without activity samples.

the vertex by two minimum ionizing particles, and a high-energy tail is mainly due to the low-energy proton. Events with energy deposition greater than $10 \mathrm{MeV}$ are considered to have activity at the vertex.

Four sub-samples, the one-track events, $\mu+p$ events, $\mu+\pi$ events with vertex activity, and $\mu+\pi$ events without vertex activity are used for constraining systematic uncertainties in the simulation. The MC distributions of the square of the four-momentum transfer $\left(Q^{2}\right)$ are fitted to the distributions of the four aforementioned data samples. The reconstructed $Q^{2}$ is calculated as

$$
Q_{\mathrm{rec}}^{2}=2 E_{\nu}^{\mathrm{rec}}\left(E_{\mu}-p_{\mu} \cos \theta_{\mu}\right)-m_{\mu}^{2},
$$

where $E_{\nu}^{\text {rec }}$ is the reconstructed neutrino energy calculated by assuming charged current quasi-elastic (CC-QE) kinematics,

$$
E_{\nu}^{\mathrm{rec}}=\frac{1}{2} \frac{\left(m_{p}^{2}-m_{\mu}^{2}\right)-\left(m_{n}-V\right)^{2}+2 E_{\mu}\left(m_{n}-V\right)}{\left(m_{n}-V\right)-E_{\mu}+p_{\mu} \cos \theta_{\mu}}
$$

where $m_{p}$ and $m_{n}$ are the mass of proton and neutron, respectively, and $V$ is the nuclear potential, which is set to $27 \mathrm{MeV}$. The fitting is described in detail in ref. [4]. Figure 4 shows the reconstructed $Q^{2}$ distributions after the fitting.

Charged current coherent pion candidates are extracted from the $\mu+\pi$ events which do not have vertex activity. The sample still contains CC-QE events in which a proton 


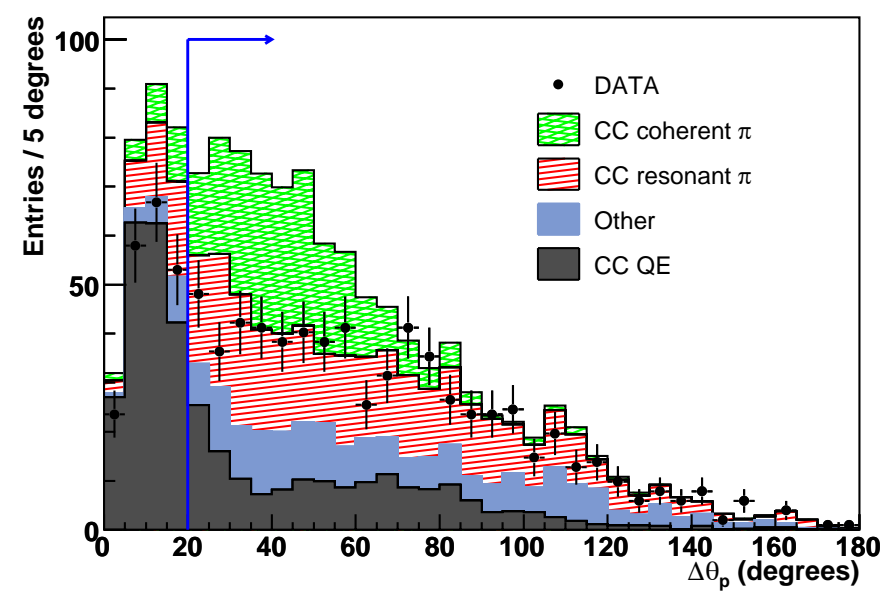

Fig. 5. $-\Delta \theta_{p}$ for the $\mu+\pi$ events in the MRD stopped sample.

is misidentified as a minimum ionizing track. We reduce this background by using kinematic information in the event. Since the CC-QE interaction is a two-body interaction, one can predict the proton direction from the measured muon momentum and muon angle. For each two-track event, we define an angle called $\Delta \theta_{p}$ as the angle between the expected proton track and the observed second track directions. Figure 5 shows the $\Delta \theta_{p}$ distribution for $\mu+\pi$ events in the MRD stopped sample. Events with $\Delta \theta_{p}$ larger than 20 degrees are selected. With this selection, $48 \%$ of charged current quasi-elastic events in the $\mu+\pi$ sample are rejected, while $91 \%$ of charged current coherent pion events pass the cut according to the MC simulation. Further selections are applied in order to separate charged current coherent pion events from charged current resonant pion events which are the dominant backgrounds for this analysis. Figure 6 shows the angular distribution of pion candidates with respect to the beam direction. In the case of charged current coherent pion events, both the muon and pion tracks are directed forward, and therefore events in which the track angle of the pion candidate with respect to the beam direction is less than 90 degrees are selected.

Figure 7 (left) shows reconstructed $Q^{2}$ for the $\mu+\pi$ events in the MRD stopped sample after the pion track direction cut. Although a CC-QE interaction is assumed, the $Q^{2}$ of charged current coherent pion events is reconstructed with a resolution of $0.016(\mathrm{GeV} / c)^{2}$ and a shift of $-0.024(\mathrm{GeV} / c)^{2}$ according to the MC simulation. Finally, events with reconstructed $Q^{2}$ less than $0.1(\mathrm{GeV} / c)^{2}$ are selected. In the signal region, 247 charged current coherent pion candidates are observed, while the expected number of background events is $228 \pm 12$. The error comes from the errors on the fitting parameters. The selection efficiency for the signal is estimated to be $10.4 \%$. The mean neutrino beam energy for true charged current coherent pion events in the sample is estimated to be 1.1 GeV after accounting for the effects of the selection efficiency.

The same selection is applied to the MRD penetrated sample to extract charged current coherent pion candidates at higher energy. Figure 7 (right) shows reconstructed $Q^{2}$ for the MRD penetrated charged current coherent pion sample. In the signal region, 57 charged current coherent pion candidates are observed, while the expected number of 


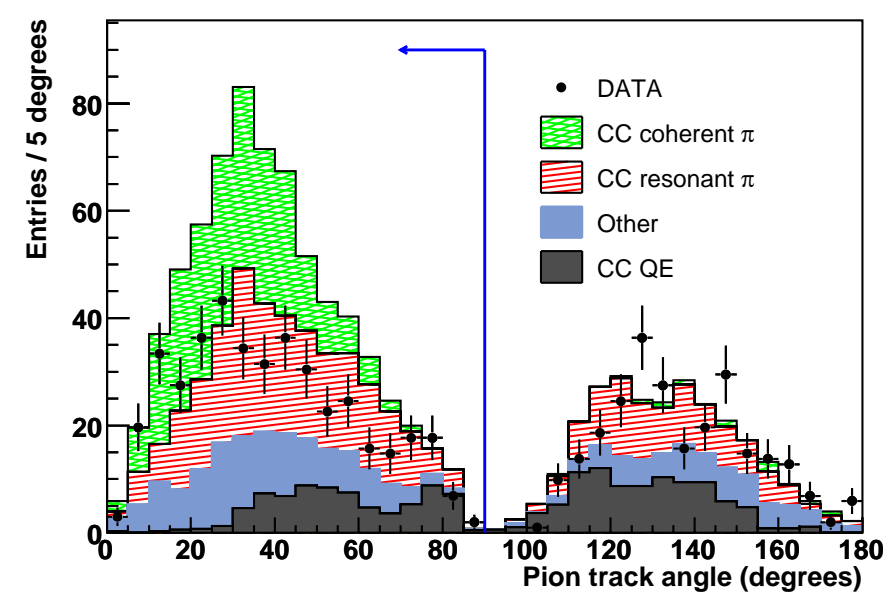

Fig. 6. - Track angle of the pion candidate with respect to the beam direction for the $\mu+\pi$ events after the charged current quasi-elastic rejection.

background events is $40 \pm 2.2$. The selection efficiency for the signal is estimated to be $3.1 \%$. The mean neutrino beam energy for true charged current coherent pion events in the sample is estimated to be $2.2 \mathrm{GeV}$.

\section{4. $-\sigma(\mathbf{C C}$ coherent $\pi) / \sigma(\mathbf{C C})$ cross-section ratio}

We measure the cross-section ratios of charged current coherent pion production to total charged current interaction with two distinct data samples. With the MRD stopped sample, the ratio of the charged current coherent pion production to total charged current cross-sections is measured to be $(0.16 \pm 0.17 \text { (stat })_{-0.27}^{+0.30}($ sys $\left.)\right) \times 10^{-2}$. The result is consistent with the nonexistence of charged current coherent pion production, and hence we set an upper limit on the cross-section ratio by using the likelihood distribution $(\mathcal{L})$ which is convolved with the systematic error. We calculate the $90 \%$ confidence level (CL)
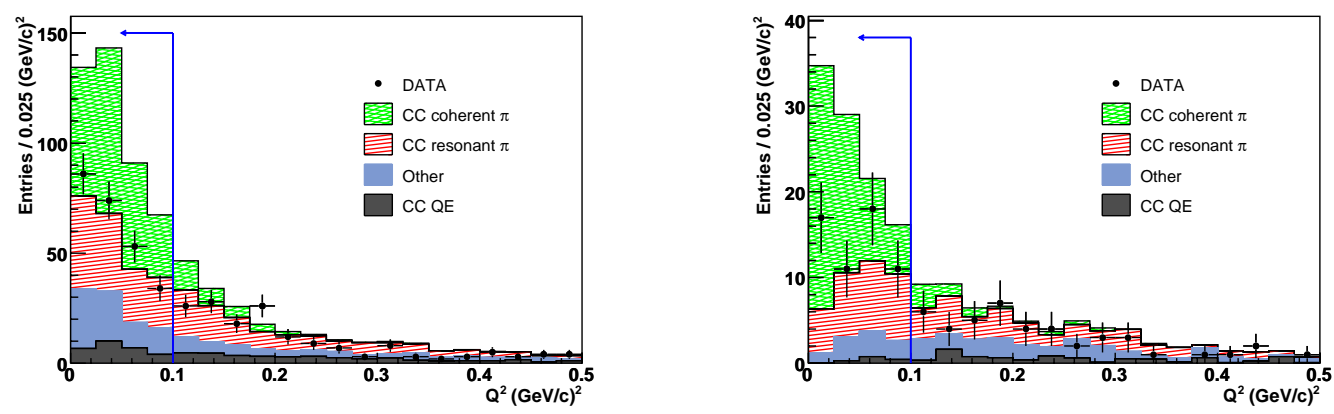

Fig. 7. - Reconstructed $Q^{2}$ for the MRD stopped charged current coherent pion sample (left), and the MRD penetrated charged current coherent pion sample (right). 
upper limit (UL) using the relation $\int_{0}^{U L} \mathcal{L} \mathrm{d} x / \int_{0}^{\infty} \mathcal{L} \mathrm{d} x=0.9$ to be

$$
\sigma(\mathrm{CC} \text { coherent } \pi) / \sigma(\mathrm{CC})<0.67 \times 10^{-2}
$$

at a mean neutrino energy of $1.1 \mathrm{GeV}$.

With the MRD penetrated sample, the cross-section ratio is measured to be $(0.68 \pm$ $0.32(\text { stat })_{-0.25}^{+0.39}($ sys $\left.)\right) \times 10^{-2}$. No significant evidence for charged current coherent pion production is observed, and hence we set an upper limit on the cross-section ratio at 90\% CL:

$$
\sigma(\mathrm{CC} \text { coherent } \pi) / \sigma(\mathrm{CC})<1.36 \times 10^{-2}
$$

at a mean neutrino energy of $2.2 \mathrm{GeV}$.

According to the Rein-Sehgal model $[5,6]$ implemented in our simulation, the cross-section ratio of charged current coherent pion production to total charged current interactions is expected to be $2.04 \times 10^{-2}$. Our limits correspond to $33 \%$ and $67 \%$ of the prediction at $1.1 \mathrm{GeV}$ and $2.2 \mathrm{GeV}$, respectively. Our results are consistent with the $\mathrm{K} 2 \mathrm{~K}$ result $[1]: \sigma(\mathrm{CC} \operatorname{coherent} \pi) / \sigma(\mathrm{CC})<0.60 \times 10^{-2}$ at $90 \% \mathrm{CL}$ measured in a $1.3 \mathrm{GeV}$ wideband neutrino beam.

\section{5. - Conclusions}

In summary, we have searched for muon neutrino charged current coherent pion production on carbon in the few-GeV region using the full SciBooNE neutrino data set of $0.99 \times 10^{20}$ POT. No evidence of charged current coherent pion production is found, and hence we set $90 \%$ CL upper limits on the cross-section ratio of charged current coherent pion production to total charged current cross-sections at $0.67 \times 10^{-2}$ and $1.36 \times 10^{-2}$, at mean neutrino energies of $1.1 \mathrm{GeV}$ and $2.2 \mathrm{GeV}$, respectively.

The SciBooNE Collaboration gratefully acknowledges support from various grants, contracts and fellowships from the MEXT (Japan), the INFN (Italy), the Ministry of Education and Science and CSIC (Spain), the STFC (UK), and the DOE and NSF (USA). The author is grateful to the Japan Society for the Promotion of Science for support.

\section{REFERENCES}

[1] Hasegawa M. et al. (K2K Collaboration), Phys. Rev. Lett., 95 (2005) 252301 [arXiv:hep-ex/0506008].

[2] Aguilar-Arevalo A. A. et al. (MiniBoone Collaboration), Phys. Lett. B, 664 (2008) 41 [arXiv:0803.3423 [hep-ex]].

[3] Aguilar-Arevalo A. A. et al. (SciBoone Collaboration), arXiv:hep-ex/0601022.

[4] Hiraide K. et al. (SciBoone Collaboration), Phys. Rev. D, 78 (2008) 112004 [arXiv:0811.0369 (hep-ex)].

[5] Rein D. and Sehgal L. M., Nucl. Phys. B, 223 (1983) 29.

[6] Rein D. and Sehgal L. M., Phys. Lett. B, 657 (2007) 207 [arXiv:hep-ph/0606185]. 